\title{
Single ventricle i-simulation
}

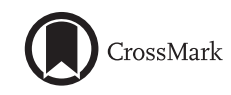

\author{
Scott M. Bradley, MD
}

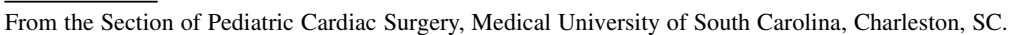

Disclosures: Author has nothing to disclose with regard to commercial support.

Received for publication Oct 24, 2017; accepted for publication Oct 26, 2017; available ahead of print Dec 7, 2017.

Address for reprints: Scott M. Bradley, MD, Pediatric Cardiac Surgery, Medical University of South Carolina,

CSB 424, 96 Jonathan Lucas St, Charleston, SC 29425 (E-mail: bradlesm@musc.edu).

J Thorac Cardiovasc Surg 2018;155:724-5

$0022-5223 / \$ 36.00$

Copyright (c) 2017 by The American Association for Thoracic Surgery

https://doi.org/10.1016/j.jtcvs.2017.10.109

Mathematical modeling of the circulatory system in patients with a single ventricle now has a 25 -year history. Some of the earliest work examined the relationship between pulmonary-to-systemic flow ratio and systemic oxygen delivery. ${ }^{2}$ More complex modeling followed soon thereafter. Computational fluid dynamic (CFD) methods were used by de Leval $^{3}$ to analyze energy dissipation and flow distribution in the total cavopulmonary connection. CFD modeling focuses on detailed local (surgical site) hemodynamic phenomena. It is carried out in 3 dimensions, and is computer resource intensive. In contrast, hydraulic lumped-parameter network modeling (LPM) allows the examination of global, system-level phenomena by dividing the circulation into interconnecting departments-it is essentially the electrical circuit diagram of the circulation. LPM is zero-dimensional and far less resource intensive than $\mathrm{CFD}$. Multiscale modeling merges information from both CFD and LPM to provide a comprehensive picture of both local hemodynamic parameters and the overall circulation. Multiscale modeling has now been used to generate sophisticated models of the entire cardiovascular system, which have shed light on many aspects of single-ventricle circulation. However, these models require specialized computer software, and as a consequence have been largely inaccessible to clinical caregivers.

The Modeling of Congenital Heart Alliance (MOCHA) is a group of 4 American and 3 European clinical and engineering institutions that have collaborated since 2010 . From 2010 to 2016, their work was funded by Fondation Leducq, a private foundation in France. The MOCHA collaboration has been quite productive, with its investigators publishing more than 60 articles in both engineering and clinical journals. The main focus of the MOCHA has been to use advanced mathematical and engineering methods, in particular multiscale modeling, to provide insights into the unique physiology of single-ventricle circulation. One of the group's primary aims has been to develop a tool that can predict postoperative hemodynamic parameters, based on patient-specific data, for staged singleventricle palliation.

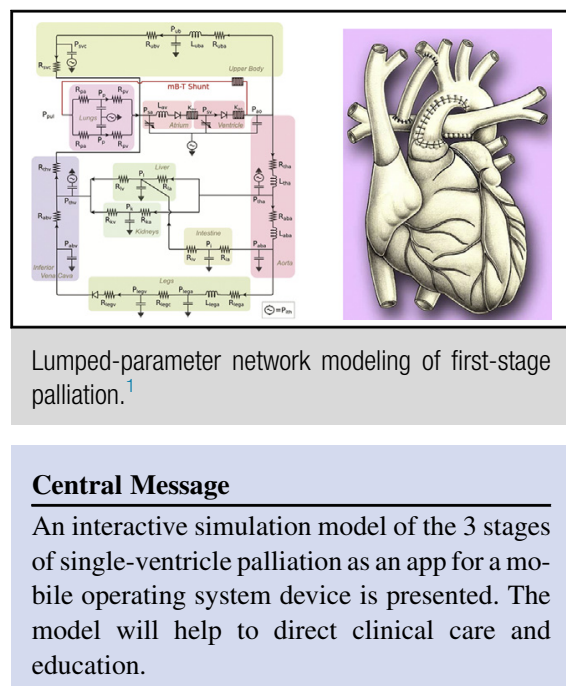

See Article page 712
In this issue, the MOCHA group builds on its extensive previous work to present a simulation model of the 3 stages of surgical single-ventricle palliation. ${ }^{1}$ The model is based on the LPM approach, so it is less computer resource intensive than CFD and multiscale modeling, and can be run on a handheld device. The model is interactive, designed to allow any interested user to enter data from a specific patient to derive outputs for that patient. The entry data include circulation type and a range of patient-specific variables. The outputs include hemodynamic variables such as systemic oxygen saturation, pulmonary-to-systemic flow ratio (Qp:Qs), pulmonary artery pressure, and mixed venous oxygen saturation. The model allows for incorporation of both venovenous and aortopulmonary collateral flow, as well as a fenestration in the Fontan pathway. The amount of fenestration flow is determined by the transpulmonary gradient and the size of the fenestration. The model does not currently incorporate atrioventricular or aortic valve regurgitation.

The results of the article consist of validation of the model by comparing simulator outputs to cardiac catheterization findings from 20 patients at each of the 3 surgical stages. ${ }^{1}$ It is important to note that the catheterization data were obtained late after a given operation, generally just before the next stage of palliation: modified BlalockTaussig shunt data before the bidirectional Glenn operation, bidirectional Glenn data before Fontan operation, and Fontan data late after Fontan surgery. The catheterizations were usually carried out under anesthesia and positive pressure 
ventilation. This means that in its current iteration, the model has been validated to accurately predict the physiologic state that exists late after surgery, under anesthesia, and on a ventilator. The model can be used to simulate a surgical stage. In the accompanying video, stage I circulation with a Blalock-Taussig shunt is modified to a bidirectional Glenn, meaning second-stage surgery was carried out. However, the data obtained for the bidirectional Glenn state reflects catheterization data obtained several years later, at the pre-Fontan catheterization. It would obviously be of great interest to predict postoperative physiology in an intensive care unit early after an operation, when the physiologic effects of cardiopulmonary bypass, myocardial ischemia/reperfusion, and acute volume shifts are in play. It is possible that the current model can indeed do this; however, validation would require collection of a large amount of data in intensive care units to compare with the simulator output.

It would also be of interest to model the response to specific management interventions, such as vasoactive medication administration, blood transfusion, extubation, and the like. Clinical reality limits the simulator to qualitative rather than quantitative analysis in this area. To quantitate the effects of a given intervention, one would need to know the patient-specific response to the intervention to enter the data into the simulator. For example, the general effects of milrinone could be simulated by increasing contractility and decreasing systemic vascular resistance. However, the quantitative patient response to milrinone (ie, how much increase in contractility and how much decrease in systemic vascular resistance) is not known, so could not be entered. The simulator can only give an estimate of the effect of any clinical intervention that generates patient-specific responses.

The primary aim of the simulator is to increase the accessibility of the model to clinicians. At the current time, the model exists as an app that runs on devices running Apple (Cupertino, Calif) mobile operating system devices, but is not available to the public. The authors made the app available to me, so I can attest that it is straightforward (and fun) to use, along the lines of the video provided in their article. It is the authors' aim to make the model available to the public via the Web. It is their hope that before final release, the model can be refined and adjusted based on multi-institutional validation involving a large number of patients. It appears that this simulator will be a useful adjunct to our current clinical decision making. It will provide a valuable education tool for caregivers; families; and, in particular, trainees. The current article ${ }^{1}$ is an important step toward bridging the existing gap between computer engineers and clinicians. We look forward to the release of the Single Ventricle i-Simulator!

\section{References}

1. Conover T, Hlavacek AM, Migliavacca F, Kung E, Dorfman A Figliola RS, et al. An interactive simulation tool for patient-specific decision support in single ventricle physiology. J Thorac Cardiovasc Surg. 2018;155:712-21.

2. Barnea O, Austin EH, Richman B, Santamore WP. Balancing the circulation: theoretic optimization of pulmonary/systemic flow ratio in hypoplastic left heart syndrome. J Am Coll Cardiol. 1994;24:1376-81.

3. De Leval MR, Dubini G, Migliavacca F, Jalali H, Camporini G, Redington A, et al. use of computational fluid dynamics in the design of surgical procedures: application to the study of competitive flows in cavo-pulmonary connections. J Thorac Cardiovasc Surg. 1996;111:502-13. 